\title{
Electrical fixing of photorefractive holograms in $\mathrm{Sr}_{0.75} \mathrm{Ba}_{0.25} \mathrm{Nb}_{2} \mathrm{O}_{6}$
}

\author{
Yong Qiao, Sergei Orlov, and Demetri Psaltis \\ Department of Electrical Engineering, California Institute of Technology, Pasadena, California 91125
}

Ratnakar R. Neurgaonkar

Rockwell International Science Center, 1049 Camino dos Rios, Thousand Oaks, California 91360

Received February 1, 1993

\begin{abstract}
Photorefractive holograms stored in $\mathrm{Sr}_{0.76} \mathrm{Ba}_{0.25} \mathrm{Nb}_{2} \mathrm{O}_{6}$ crystals are electrically fixed at room temperature. The fixed holograms can be read out directly or after a positive-voltage pulse is applied that can dramatically enhance the diffraction efficiency. Single gratings as well as images are recorded and fixed.
\end{abstract}

Volume holograms recorded in photorefractive materials can find important applications in optical memories and optical computing systems. One problem with a photorefractive hologram is that it gets erased by the readout light. Nondestructive readout can be achieved by hologram fixing, and several fixing methods have been reported. Thermal fixing of holograms was demonstrated in $\mathrm{LiNbO}_{3},{ }^{1}$ $\mathrm{Bi}_{12} \mathrm{SiO}_{20},{ }^{2} \mathrm{KNbO}_{3},{ }^{3}$ and $\mathrm{BaTiO}_{3},{ }^{4}$ where a compensating ionic charge grating (which cannot be erased optically) is formed at an elevated crystal temperature. Micheron and Bismuth demonstrated hologram fixing in $\mathrm{Sr}_{0.75} \mathrm{Ba}_{0.25} \mathrm{Nb}_{2} \mathrm{O}_{6}$ (Ref. 5; SBN:75) and $\mathrm{BaTiO}_{3}$ (Ref. 6) through the creation of a ferroelectric domain pattern by applying an external field at room temperature. Hologram fixing in SBN:75 was also achieved by cooling the exposed crystal through the ferroelectric phase transition. ${ }^{7}$ Leyva et al. demonstrated hologram fixing in $\mathrm{KTa}_{1-x} \mathrm{Nb}_{x} \mathrm{O}_{3}$ by cooling the exposed crystal under an applied field through the ferroelectric phase transition. ${ }^{8}$ In general, electrical fixing is preferable from a practical point of view because of its relative simplicity.

In this Letter we report the results of our investigation on electrical fixing of photorefractive holograms recorded in SBN:75. We were able to reproduce some of the effects that Micheron and Bismuth reported in Ref. 5, but our observations were different in several important respects. In addition, we report two novel ways of electrically fixing holograms in SBN:75 that give improved performance and demonstrated that holograms of images can be fixed and faithfully reproduced.

The crystal sample used in the experiment was grown and poled at Rockwell International Science Center. It has dimensions of $6 \mathrm{~mm} \times 6 \mathrm{~mm} \times 6 \mathrm{~mm}$, with its $c$ axis parallel to the edges. An eternal electric field can be applied along the $c$ axis, and it is called positive (negative) if its direction is same as (opposite) that of the initial poling filed. In our experimental setup (Fig. 1), an ordinary-polarized plane wave from an argon laser $(\lambda=488 \mathrm{~nm})$ is split into three beams, two of which are used for recording a grating in the crystal, with the third used as a nonBragg-matched erasing beam. The grating vectors are approximately parallel to the $c$ axis and the total recording intensity is $\sim 10 \mathrm{~mW} / \mathrm{cm}^{2}$. The diffraction efficiency $\eta$ is monitored with a low-intensity, extraordinary-polarized $\mathrm{He}-\mathrm{Ne}$ laser beam incident at the Bragg angle. The diffraction efficiency is calculated by subtracting the background noise level from the measured diffracted light and dividing the difference by the transmitted light power.

In the first experiment, a holographic grating with a grating spacing $\Lambda=11.6 \mu \mathrm{m}$ was recorded in the completely poled crystal without any applied field. After the diffraction efficiency $\eta$ reached its saturation value $(\eta=11 \%)$, the recording beams were blocked, and a negative-voltage pulse with amplitude $V=-1 \mathrm{kV}$ and duration $t=0.5 \mathrm{~s}$ was applied to the crystal, which caused $\eta$ to fall quickly. After the voltage pulse was removed, $\eta$ recovered a portion of its initial value before the pulse. Then the crystal was illuminated with the non-Bragg-matched erasing beam, and $\eta$ decreased further until it reached a steady-state value of $\eta \approx 0.06 \%$. This fixed grating could not be erased by the erasing beam. Then the erasing beam was blocked, and a positive-voltage pulse, with amplitude $V=+2 \mathrm{kV}$ and duration of a

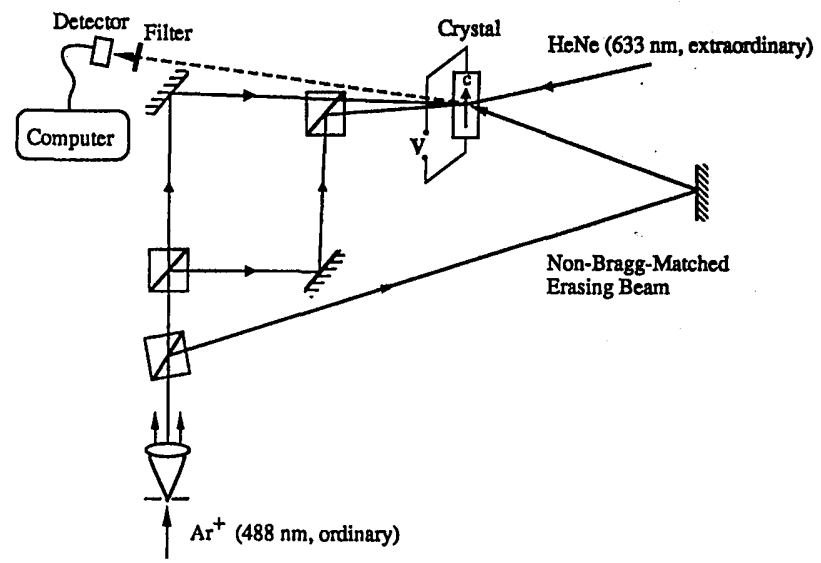

Fig. 1. Optical setup. 


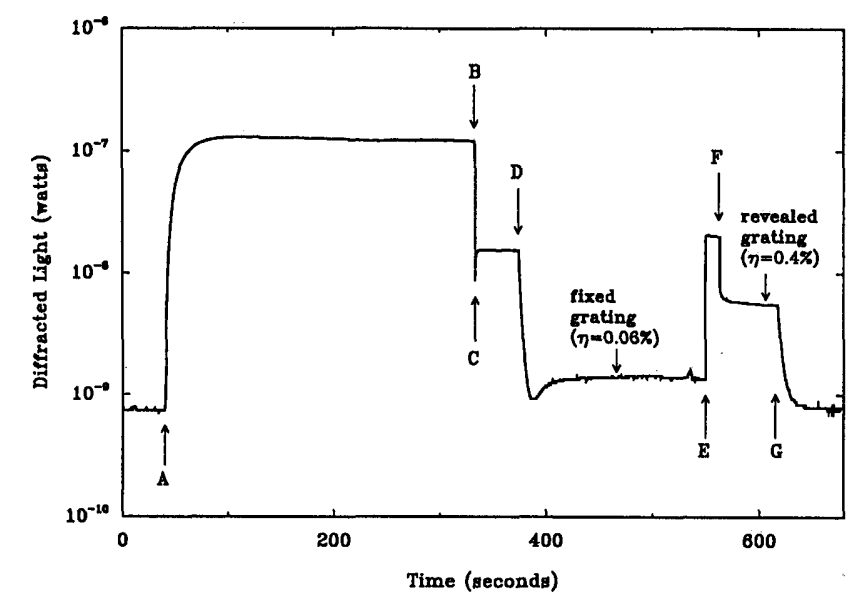

Fig. 2. Diffracted light as a function of time for fixing with a negative-voltage pulse $(\Lambda=11.6 \mu \mathrm{m})$. The transmitted light power in the absence of the grating is $1.1 \mu \mathrm{W}$. A, Hologram recording begins. $B$, Negative pulse is applied. C, End of negative pulse. D, Optical erasure begins. E, Erasing beam is blocked and positivevoltage pulse is applied. F, End of positive pulse. G, Optical erasure begins.

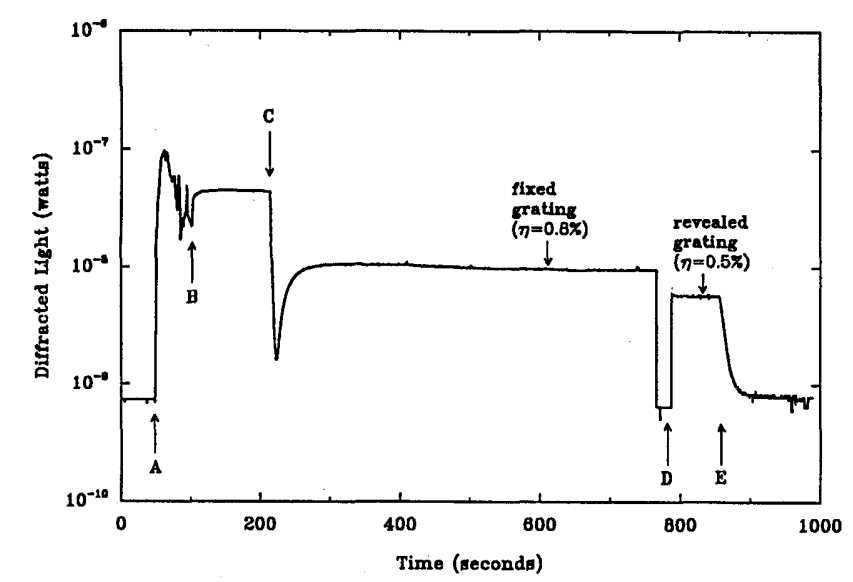

Fig. 3. Diffracted light as a function of time for fixing with a constant negative voltage during recording $(\Lambda=11.6 \mu \mathrm{m})$. The transmitted light power in the absence of the grating is $1.1 \mu \mathrm{W}$. A, Hologram recording begins and negative voltage is applied. B, Negative voltage is removed and recording beams are blocked. $\mathrm{C}$, Optical erasure begins. D, Positive-voltage pulse is applied (probe beam is blocked). E, Optical erasure begins.

few seconds, was applied to the crystal. During the positive-voltage pulse, $\eta=1.8 \%$, and after the pulse a grating with $\eta=0.4 \%$ was revealed. This revealed grating can be optically erased. The experimental results of recording, fixing, revealing, and erasing are shown in Fig. 2, where the diffracted signal is plotted as a function of time during the different stages of the experiment.

Comparing the results in Fig. 2 with Ref. 5, we see that we were "able to achieve electrical fixing, but the fixed grating obtained in our experiment is much weaker than that in Ref. 5. Another important difference is our observation of a revealed grating on application of a strong positive voltage across the crystal at relatively large grating spacings.
We have found that in general the strengths of the fixed grating and the revealed grating can be greatly enhanced by applying a constant negative voltage during recording. With an applied voltage of $V=-500 \mathrm{~V}$, we recorded a grating with $\Lambda=$ $11.6 \mu \mathrm{m}$, using an exposure time of $50 \mathrm{~s}$. Then the recording beams were blocked, the applied voltage was removed, and the grating was allowed to settle down to a steady level. On illumination of the crystal with the erasing beam, $\eta$ decreased first and then rose back to a steady state, indicating a fixed grating of $\eta=0.8 \%$. Finally, a positive-voltage pulse, the same as the one used in the previous experiment, was applied, which revealed a grating of $\eta=0.5 \%$. This experimental result is shown in Fig. 3.

The experiment described above was repeated for several different grating spacings, and the results are summarized in Fig. 4. Under our experimental conditions, the maximum diffraction efficiency of the fixed grating is found to be $0.8 \%$ at $\Lambda=$ $11.6 \mu \mathrm{m}$. The revealed grating is observed only for relatively large $\Lambda$, and its strength increases drastically with the increase in $\Lambda$, achieving approximately $17 \%$ diffraction efficiency at $\Lambda=29 \mu \mathrm{m}$.

Finally, an image was recorded in the crystal as an image plane hologram, and Fig. 5(a) shows the reconstruction of the hologram. Figure $5(\mathrm{~b})$ shows the reconstruction of the fixed hologram. The fixed hologram did not show any sign of degradation un-
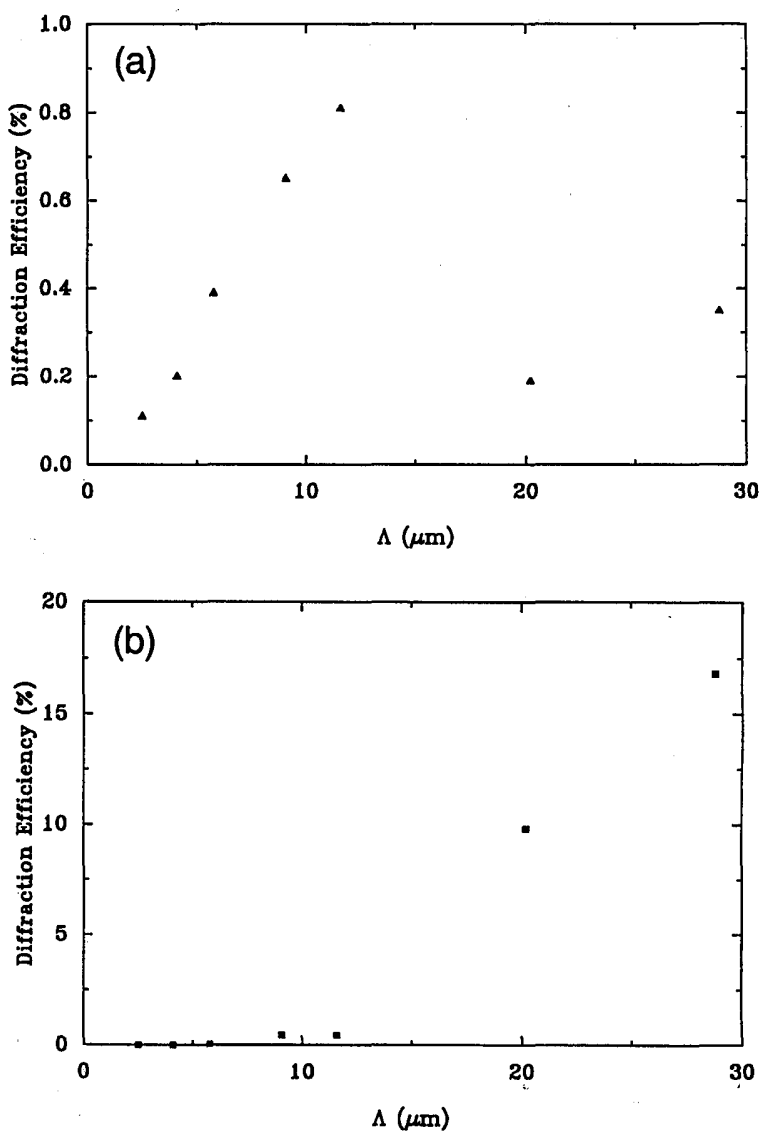

Fig. 4. Diffraction efficiency as a function of grating spacing for (a) the fixed grating and (b) the revealed grating. 


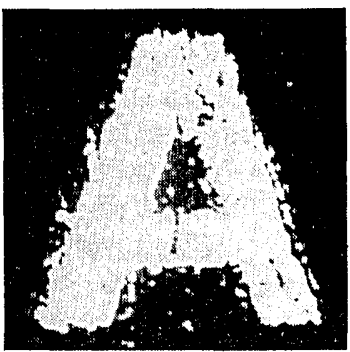

(a)

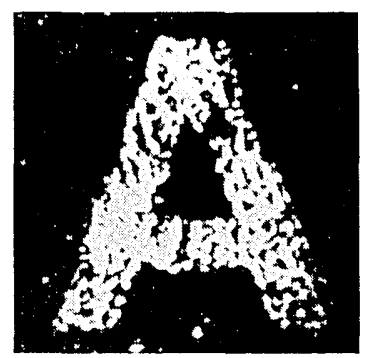

(b)
Fig. 5. (a) Reconstruction of the recorded hologram and (b) reconstruction of the fixed hologram.

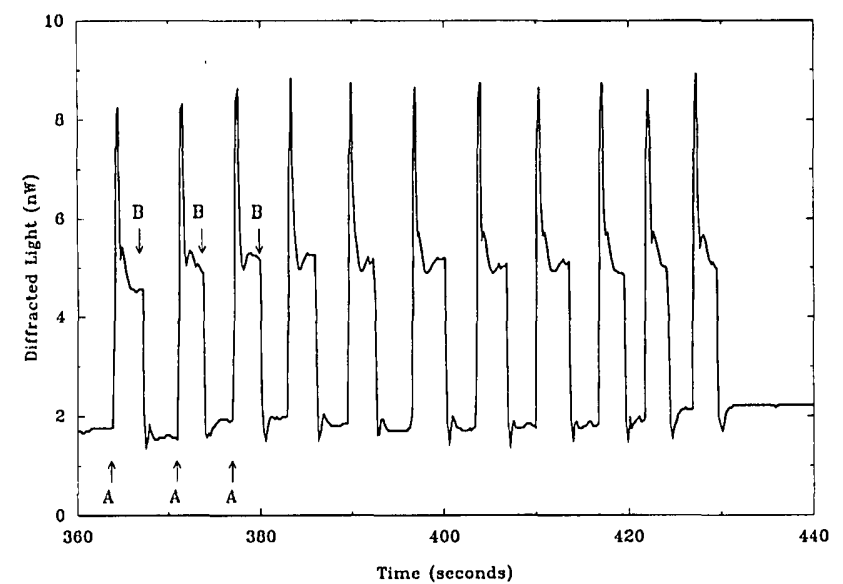

Fig. 6. Cyclic fixing and revealing of the stored hologram ( $\Lambda=20.2 \mu \mathrm{m}$ ), with the erasing beam off during the entire process. A, Positive (revealing) pulse is applied, with $V=+1 \mathrm{kV}$. B, Negative (fixing) pulse is applied, with $V=-1 \mathrm{kV}$.

der illumination with the non-Bragg-matched erasing beam for $2.5 \mathrm{~h}$.

The fixing-and-revealing process that we described was not observed when a positive rather than a negative fixing voltage was applied. If we assume that the mechanism responsible for fixing involves compensating ions, then we have no obvious explanation for the asymmetry regarding the polarity of the fixing voltage. Similarly, if the mechanism involved two types of photorefractive species, ${ }^{9}$ the effect would not be sensitive to the polarity of the fixing voltage. The fact that the fixed grating can be electrically erased nearly instantaneously (less than $0.1 \mathrm{~s}$ ) in the dark (i.e., without redistribution of charge carriers among the trap sites). also indicates that the effect does not involve two types of trap site that compensate for each other. Therefore we believe that the mechanism responsible for this effect is the polarization grating formation suggested in Ref. 5 .

Specifically, our observations can be explained as follows. With the aid of the negative external field, the electronic space-charge field established during the holographic recording causes a spatial modulation of the ferroelectric polarization. In the areas where the space-charge field is negative, the local polarization is modified (which corresponds to local depoling or possible repoling in the opposite direction). This causes the decrease of both the mean linear electro-optic coefficient and the amplitude of the space-charge field modulation. As a result, the amplitude of the refractive-index modulation decreases on application of a negative voltage pulse, causing the drop in the monitored diffraction efficiency. Under the illumination of the erasing beam, the electronic grating is erased further, until a balance between the polarization grating and the electronic grating is reached. The fixed grating observed is attributed to the polarization grating for which the electronic grating partially compensates. Finally, a strong positive voltage applied to the crystal erases the polarization grating (i.e., the ferroelectric domains are realigned), revealing the compensating electronic grating, which of course can be erased optically. The enhancement of the diffraction efficiency of the revealed grating during the positivevoltage pulse (see Fig. 2) is attributed to the nonlinear electro-optic effect in SBN:75. ${ }^{10}$ We have developed a physical model that describes the above process, and the predicted dependence of the diffraction efficiencies on the grating spacing closely matches our experimental observations. ${ }^{11}$

The above experiments suggest two modes of hologram fixing in SBN:75 crystals: the fixed polarization grating and the revealed electronic grating. Although the revealing process is destructive to the polarization grating, it is possible to recreate this grating by applying a negative-voltage pulse after the electronic grating is revealed. These revealing/fixing cycles were repeatedly performed (shown in Fig. 6), and the diffraction efficiency of the revealed grating (and thus the polarization grating) remained unchanged.

This research is supported by the Defense Advanced Research Projects Agency and the U.S. Air Force Office of Scientific Research. We thank WhyeKei Lye for his help.

\section{References}

1. J. J. Amodei and D. L. Staebler, Appl. Phys. Lett. 18, 540 (1971)

2. L. Arizmendi, J. Appl. Phys. 65, 423 (1989).

3. G. Montemezzani and P. Günter, J. Opt. Soc. Am. B 7, 2323 (1990).

4. D. Kirillov and J. Feinberg, Opt. Lett. 16, 1520 (1991).

5. F. Micheron and G. Bismuth, Appl. Phys. Lett. 23, 71 (1973).

6. F. Micheron and G. Bismuth, Appl. Phys. Lett. 20, 79 (1972).

7. F. Micheron and J. Trotier, Ferroelectrics 8, 441 (1974).

8. V. Leyva, A. Agranat, and A. Yariv, Opt. Lett. 16, 554 (1991).

9. G. S. Trofimov and S. I. Stepanov, Sov. Tech. Phys. Lett. 10, 282 (1984).

10. J. P. Wilde and L. Hesselink, Opt. Lett. 17, 853 (1992).

11. S. Orlov, Y. Qiao, and D. Psaltis, "Dynamic compensation of tixed gratings in photorefractive media," submitted to J. Opt. Soc. Am. B. 